A N N A L E S Annales de Bretagne et des Pays de l'Ouest

\title{
Le retable anversois de la cathédrale de Rennes. Un chef-d'œuvre révélé
}

\section{Georges Provost}

\section{(2) OpenEdition}

1 Journals

\section{Édition électronique}

URL : https://journals.openedition.org/abpo/6567

DOI : $10.4000 / a b p o .6567$

ISSN : 2108-6443

\section{Éditeur}

Presses universitaires de Rennes

\section{Édition imprimée}

Date de publication : 10 décembre 2020

Pagination : $217-219$

ISBN : 978-2-7535-8225-5

ISSN : 0399-0826

Référence électronique

Georges Provost, "Le retable anversois de la cathédrale de Rennes. Un chef-d'œuvre révélé », Annales de Bretagne et des Pays de l'Ouest [En ligne], 127-4 | 2020, mis en ligne le 10 décembre 2020, consulté le 06 janvier 2023. URL : http://journals.openedition.org/abpo/6567 ; DOI : https://doi.org/10.4000/ abpo.6567 
introduction. Cette dernière remarque n'enlève rien à la qualité de l'ouvrage, qui saura intéresser bien au-delà des seuls cercles ruralistes, avec des présentations méthodologiques rigoureuses et utiles pour les historiens.

Brice RABOT

Guillot DE Suduiraut, Sophie (dir.), Le retable anversois de la cathédrale de Rennes. Un chef-d'œuvre révélé, Rennes, Presses universitaires de Rennes, 2019, 207 p.

Quelque peu incongru dans un grand vaisseau néo-classique paré d'ors et de stucs au temps de $M^{\text {gr }}$ Brossays Saint-Marc, le grand retable gothique de la cathédrale Saint-Pierre fait aujourd'hui l'objet d'une véritable redécouverte dont témoigne ce très bel ouvrage des Presses universitaires de Rennes. L'œuvre était jusqu'ici mal servie par sa disposition dans une chapelle latérale qui ne lui donnait ni lumière, ni recul; le retable se trouvait confiné derrière un écran vitré dont l'objectif de sécurité n'avait pas empêché le vol de plusieurs pièces en 2007. La restauration de l'ensemble des décors de la cathédrale, remarquablement menée depuis 2009 par l'État maître d'ouvrage, a été l'occasion de concrétiser le projet ancien de faire de cet objet exceptionnel le joyau d'un Trésor en libre accès dans l'ancienne sacristie Nord.

Pour la première fois depuis 1872 , l'occasion était ainsi donnée de déposer le retable, de le soumettre à des analyses approfondies et de le restaurer selon les principes actuellement reconnus. À la joie d'admirer cette œuvre comme jamais, le livre ajoute donc le plaisir d'accéder à tout ce que le chantier a révélé : les textes, les clichés photographiques et la séduisante maquette sont en tous points remarquables. L'ouvrage, dirigé par Sophie Guillot de Suduiraut, ancienne conservatrice en chef au département des Sculptures du musée du Louvre, a bénéficié de sa compétence en matière de retables qu'elle dit " brabançons ", qualificatif mieux accordé à la réalité politique du début du Xvi ${ }^{\mathrm{e}}$ siècle que le trop extensif " flamand ». Le livre réunit également les signatures des conservateurs des Monuments Historiques en Bretagne (Henry Masson, Christine Jablonski, Cécile Oulhen) et l'expertise technique des maîtres d'œuvre, français et belges, des études et de la restauration proprement dite.

L'origine anversoise du retable, avancée depuis les années 1950, se trouve ainsi confirmée et précisée par le repérage sur la boiserie de pas moins de 34 mains et 2 châteaux (armes d'Anvers) qui sont autant de " marques de garantie " apposées au fer. Tous les indices concordent pour dater le retable des environs de 1520. D'une part, l'analyse dendrochronologique a révélé l'origine du bois - un excellent chêne dit waghenschot, provenant de l'est de la Baltique - et en a établi l'abattage entre 1512 et 1522 . D'autre part, l'analyse proprement stylistique situe l'œuvre rennaise par rapport aux 180 retables anversois du Xvi ${ }^{\mathrm{e}}$ siècle conservés aujourd'hui du Portugal à la Suède (dont une vingtaine en France). Le retable de Rennes correspond à un certain apogée des ateliers anversois, où se conjuguent qualité des matériaux, production intensive, maîtrise du style. Celui-ci peut se définir par une élégance gracieuse, un peu affectée parfois, mais sans le maniérisme sensible à partir de 1530; sans vocabulaire italianisant encore, sinon dans un ou deux hypothétiques détails. On savait déjà, en particulier depuis la thèse de Gildas Durand, combien la Bretagne avait été réceptive à la production artistique des anciens Pays-Bas, avec lesquels la péninsule entretenait d'intenses relations commerciales. Christine Jablonski actualise ici, de façon précise, la liste des sculptures bretonnes d'impor- 
tation nordique : outre les retables anversois de Rennes et Kerdévot, près d'une dizaine de sculptures isolées, jusque dans des églises rurales de l'intérieur comme au Vieux-Bourg de Lothey. La frontière est également précisée avec des œuvres locales d'inspiration "flamande".

L'ouvrage retrace aussi, par des schémas très parlants, le remarquable exercice de " puzzle en relief " qui a permis de rétablir l'ordonnancement des scènes du retable en réintégrant des sculptures qui s'en étaient séparées au long des âges : le Mariage d'Anne et Joachim bien sûr, seule pièce retrouvée après le vol de 2007, mais aussi la Vierge de l'Adoration des Bergers (demeurée depuis le XIX ${ }^{\mathrm{e}}$ siècle au Musée des Beaux-Arts de Rennes), un membre de la suite des Mages identifié au musée de Cluny et une autre sculpture, volée en 1975, et retrouvée au musée de Riom! Nombreux sont également les personnages ou éléments de décor à avoir retrouvé leur place initiale. Aussi peut-on parler d'une véritable redécouverte : restitué dans sa polychromie originelle - en particulier les dorures, les lettres ornementales, les somptueux vêtements des personnages secondaires contrastant avec la simplicité des " saints ", les très délicates carnations qui redonnent tant de vie aux 77 visages... - le retable suscite, dans son nouvel emplacement, une perception totalement renouvelée. Et il n'est pas interdit d'espérer de futures réintégrations de complément : les autres reliefs volés en 2007 referont bien surface un jour; un second Mage semble déjà avoir été identifié, mais non localisé...

C'est que l'histoire du retable est fort complexe, et Cécile Oulhen la retrace aussi précisément que possible. Contrairement à l'hypothèse souvent retenue ces dernières années, il ne provient pas originellement de la chapelle de l'hôpital SainteAnne : s'il a pu y séjourner un temps, à la fin du xvIII ${ }^{\mathrm{e}}$ siècle, il a indéniablement été commandé pour l'ancienne cathédrale dont il fut le maître-autel. Il semble en effet mentionné dans le procès-verbal de prééminences dressé en 1755, en prévision de la démolition de l'édifice. Commence alors pour le retable anversois une séquence redoutable de démembrements (les volets peints, encore attestés en 1755, disparaissent) et de déménagements successifs : chapelle Sainte-Anne, vieille église Saint-Aubin (jusqu'en 1812, au témoignage sûr de l'abbé Carron, curé de SaintGermain), recoins de la ci-devant abbatiale Saint-Melaine (1813) puis greniers du palais épiscopal où il se trouve lorsque des membres de la Société Française pour la Conservation des Monuments historiques (future Société Française d'Archéologie) le signalent en 1840. À l'heure de la redécouverte du gothique, les années médianes du XIX ${ }^{\mathrm{e}}$ siècle voient une véritable entreprise de sauvetage conduite par Prosper Mérimée et Arcisse de Caumont, localement menée par Jules Aussant à qui le retable doit beaucoup. Comme membre fondateur puis président de la Société archéologique d'Ille-et-Vilaine, avant de devenir conservateur du Musée archéologique, il parvient à faire ressurgir bien des fragments dispersés jusqu'en Basse-Bretagne et obtient le dépôt de l'œuvre au Musée. Le retable manque pourtant de disparaître à nouveau quand $\mathrm{M}^{\mathrm{gr}}$ Brossays Saint-Marc envisage, en 1857, de le vendre pour financer les travaux de décoration de la nouvelle cathédrale. La modestie du prix de vente l'en dissuada finalement mais il tint à récupérer l'œuvre, qu'il fit installer en 1872 dans l'ancienne chapelle Saint-Melaine de sa " Métropole ".

L'arrivée du retable à Rennes n'est pas précisément documentée mais plusieurs indices attestent, aux environs de 1520, d'un mouvement cohérent de rénovation du chœur de la cathédrale : jubé et balustrade, stalles (remplacées au début des années 1520 grâce au mécénat de la famille d'Epinay) et donc retable. L'ouvrage évoque ici le possible rôle de l'évêque Yves Mahyeuc. Sur ce point, il me semble possible d'aller plus loin en se fondant sur la déclaration d'un déposant au procès en béatification de ce dernier en 1637 : Roch Lezot dit tenir de son propre père (mort en 1600) que le " bon Yves " avait " fait le chœur de ladite église élever, 
mettre en lambris et peindre toutes colonnes dudit chœur en image de Paradis et couronnement de la béate Vierge comme il paraît encore " (traduction d'Augustin Pic dans Augustin Pic et Georges Provost, dir. Yves Mahyeuc. Rennes en Renaissance, Rennes, PUR, 2010, p. 354-355). Certes, il n'est pas question ici du retable mais le choix de représenter le Couronnement de la Vierge semble significatif à deux titres. D'une part, il renforce la probabilité que le maître-autel ait été dédié à la Vierge (de manière assez inattendue dans une cathédrale sous le vocable de saint Pierre); d'autre part, la scène du Couronnement de la Vierge suggère une forme de continuité par rapport au cycle narratif du retable qui s'achève par l'Assomption. L'évêque dominicain aura ainsi voulu prendre sa part de l'embellissement du chœur de la cathédrale, en prolongement du retable dont l'initiative reviendrait plus vraisemblablement au chapitre, hypothèse que renforce aujourd'hui la qualité exceptionnelle de l'objet. Combien l'on regrette ici que les délibérations capitulaires ne soient conservées qu'à partir de 1526 !

Deux autres indices, valables pour la fin du XVII ${ }^{\mathrm{e}}$ siècle, me semblent confirmer la présence dans le chœur de la cathédrale d'un maître-autel désormais quelque peu archaïque par rapport aux dévotions tridentines. En 1687, un inventaire (Arch. dép. Ille-et-Vilaine, $1 \mathrm{G}$ 261/1) atteste en effet la présence d'une " custode d'argent continuellement suspandue sur le grand autel " (donc une suspension eucharistique) et aussi d'" un tabernacle de bois doré pour servir à exposer le Très Saint Sacrement " : ne peut-on y voir les compléments, perçus comme indispensables à la fin du XVII e siècle, à un maître-autel gothique dépourvu de tabernacle? À cette date, la priorité eucharistique donnée au maître-autel tendait sans doute à faire oublier le caractère marial des scènes représentées (d'autant que la cathédrale s'était dotée en 1635 d'un autre autel à la Vierge, dit "du Vœu ", dans le transept Nord). On s'explique mieux qu'en 1755, l'inventaire des prééminences de la cathédrale l'ait caractérisé sommairement comme " la naissance et la vie du Sauveur ".

En retrouvant sa beauté initiale, le retable anversois de la cathédrale livre ainsi une part de ses mystères. Il confirme la réalité de cette "Renaissance rennaise " d'avant 1532, si peu italienne encore mais pas moins "renaissante " pour autant. Et il avive notre attente du livre de synthèse sur la cathédrale Saint-Pierre, entreprise collective en cours depuis plusieurs années mais dont l'heureux aboutissement, sous la direction de Jean-Yves Andrieux, paraît désormais en vue.

Georges Provost

Alain PAuQuet, Villageois en Touraine. La société à Chédigny de 1590 à 1914, Chemillé-sur-Indrois, Éditions Hugues de Chivré, 2020, 383 p.

Avec cet ouvrage, Alain Pauquet, agrégé d'histoire et docteur ès-lettres, nous offre une très belle étude d'histoire rurale. Si aucun reproche ne peut être fait à l'édition - la présentation et la réalisation du livre sont en effet excellentes -, il faut dire d'emblée que le travail de recherche très fouillé auquel s'est livré l'auteur aurait sans doute davantage trouvé son public s'il avait été proposé et diffusé par un éditeur national (ou universitaire). En effet, contrairement à ce que pourrait laisser supposer son titre, le livre d'Alain Pauquet est bien plus qu'une " classique monographie communale ". L'auteur, spécialiste de l'histoire politique et sociale du pays, est du reste un chercheur chevronné. Ancien élève de Maurice Aguhlon, Alain Pauquet a consacré sa thèse à la société en Berry au milieu du XIX ${ }^{\mathrm{e}}$ siècle, thèse qu'il a soutenue en 1993 à Paris 1 Panthéon-Sorbonne et publiée en 1998 aux éditions 